\title{
Integrated Model Reduction and Control of Aircraft with Flexible Wings
}

\author{
Sean Shan-Min Swei* , Guoming G. Zhu ${ }^{\dagger}$, Nhan Nguyen ${ }^{\ddagger}$
}

\begin{abstract}
This paper presents an integrated approach to the modeling and control of aircraft with flexible wings. The coupled aircraft rigid body dynamics with a high-order elastic wing model can be represented in a finite dimensional state-space form. Given a set of desired output covariance, a model reduction process is performed by using the weighted Modal Cost Analysis (MCA). A dynamic output feedback controller, which is designed based on the reduced-order model, is developed by utilizing output covariance constraint (OCC) algorithm, and the resulting OCC design weighting matrix is used for the next iteration of the weighted cost analysis. This controller is then validated for full-order evaluation model to ensure that the aircraft's handling qualities are met and the fluttering motion of the wings suppressed. An iterative algorithm is developed in CONDUIT environment to realize the integration of model reduction and controller design. The proposed integrated approach is applied to NASA Generic Transport Model (GTM) for demonstration.
\end{abstract}

\section{Introduction}

New energy efficient aircraft concepts are being studied by NASA, industries, and academia, through better aerodynamic design, use of light weight structures, and novel flight control concepts. In particular, lightweight aircraft design has attracted considerable attentions in recent years in an effort to improve cruise efficiency and lower the induced drag. As structural flexibility increases, aeroelastic interactions with aircraft aerodynamic forces and moments can have adverse impact on aircraft's stability and performance. More specifically, the modal frequencies of flexible wings can be in the range of aircraft's rigid body modes and within the flight control bandwidth. Therefore, designing an effective aircraft flight control law becomes a challenging task.

The aeroelastic aircraft control problem considered in this paper utilizes work of two disciplines; control of flexible structures and aircraft flight control law development. In the context of flexible structure controls, one must first develop an adequate mathematical model, which is reduced in size, prior to designing an suitable control. A number of model reduction methods ${ }^{1-5}$ can be used for carrying out the order reduction. One method that we find it most appropriate for our study is the Modal Cost Analysis. ${ }^{5}$ In MCA the total cost is expressed as a sum of modal cost of each aeroelastic mode to the defined output covariance cost function. Therefore, by examining each modal cost, we can attain a reduced-order model by retaining those modes with higher modal cost. The performance requirements for vibrational aeroelastic wings subject to random gust load can be prescribed in terms of output covariance. Since both model reduction and aeroelasticity effect use covariance as a measure of preformance, we utilize the output covariance constraint (OCC) control algorithm ${ }^{9}$ to develop an integrated modeling and control algorithm to suppress the vibrational motion of the wings. The essence of OCC problem is finding appropriate weighting matrices so that a stabilizing controller is obtained while minimizing the control effort and meeting constraints on the output covariance.

In the context of designing a flight control law, we utilize CONDUIT (CONtrol Designer's Unified Interface), ${ }^{11}$ a state-of-the-art multidisciplinary computational facility, in attaining optimal solution to the aeroelastic aircraft control problem. In CONDUIT, both handling qualities specifications for aircraft rigid body dynamics and OCC for aeroelastic wings are evaluated throughout optimization process. In this case, weighting matrices are considered design parameters to be tuned at every CONDUIT simulation. Therefore, the aeroelastic aircraft control problem, consisting of model reduction, OCC control, and flight control

*Research Scientist, Intelligent Systems Division, NASA Ames Research Center

$\dagger$ Professor, Mechanical Engineering, Electrical and Computer Engineering, Michigan State University

${ }^{\ddagger}$ Research Scientist, Intelligent Systems Division, NASA Ames Research Center 
design, can be entirely setup in CONDUIT environment. A preliminary analysis result for NASA Generic Transport Model (GTM) is presented.

\section{Coupled Aeroelastic and Aircraft Dynamics}

The coupled aircraft rigid body dynamics with $2 N$ aeroelastic wing modes; $N$ bending modes and $N$ torsional modes, can be described by

$$
\begin{aligned}
& M_{e} \ddot{x}_{e}+C_{e} \dot{x}_{e}+K_{e} x_{e}+C_{a} x_{a}=\left[\begin{array}{ll}
D_{f} & D_{s}
\end{array}\right]\left[\begin{array}{c}
\delta_{f} \\
\delta_{s}
\end{array}\right] \\
& \dot{x}_{a}=A_{a} x_{a}+A_{s} x_{e}+A_{d} \dot{x}_{e}+B_{e} \delta_{e}+\left[\begin{array}{ll}
B_{f} & B_{s}
\end{array}\right]\left[\begin{array}{c}
\delta_{f} \\
\delta_{s}
\end{array}\right]
\end{aligned}
$$

where $x_{e}=\left[w_{1} w_{2} \cdots w_{N} \theta_{1} \theta_{2} \cdots \theta_{N}\right]^{t} \in R^{2 N}$ denotes the displacement for bending and torsional flexible modes, $x_{a} \in R^{n_{a}}$ the aircraft's rigid body states, e.g. angle of attack and pitch rate for short-period mode, $\delta_{e}$ denotes the elevator/aileron/rudder deflection angle, $\delta_{f}$ and $\delta_{s}$ denote, respectively, flap and slat deflections. The size of $x_{e}$ can be very large, hence we consider the model given in (1) be a true enough model and will be used to evaluate the designed controllers.

It is understood that the triple matrices $\left(M_{e}, C_{e}, K_{e}\right)$ in (1) do not have the usual structural properties, that is, they are neither symmetric nor sign definite. It is apparent from (1) that the aircraft rigid body dynamics and the aeroelastic modes are coupled. When the couplings are neglected, the performance of flexible modes and aircraft open-loop behavior can be determined by examining the triple matrices $\left(M_{e}, C_{e}, K_{e}\right)$ and the eigenvalues of $A_{a}$. We regard this as "uncoupled"' condition, which shows some underline system characteristics. The detail derivations and physical interpretations of (1) can be found, for instance, in Ref. 6.

For better illustration, we rewrite (1) in a state-space representation form as follows,

$$
\dot{x}_{p}=A_{p} x_{p}+B_{p} u_{\delta},
$$

where $x_{p}=\left(x_{e}, \dot{x}_{e}, x_{a}\right)$ and $u_{\delta}=\left(\delta_{e}, \delta_{f}, \delta_{s}\right)$, and

$$
A_{p}=\left[\begin{array}{ccc}
0 & I & 0 \\
-M_{e}^{-1} K_{e} & -M_{e}^{-1} C_{e} & -M_{e}^{-1} C_{a} \\
A_{s} & A_{d} & A_{a}
\end{array}\right] ; B_{p}=\left[\begin{array}{ccc}
0 & 0 & 0 \\
0 & M_{e}^{-1} D_{f} & M_{e}^{-1} D_{s} \\
B_{e} & B_{f} & B_{s}
\end{array}\right] .
$$

We assume that the pair $\left(A_{p}, B_{p}\right)$ is controllable. In this paper, we will consider modal reduction process for flexible wings, hence it makes little practical sense if the residual modes are unstable. Therefore, we assume $A_{p}$ is Hurwitz and has distinctive eigenvalues.

\section{II.A. Wind Turbulence Model}

We consider the coupled aeroelastic aircraft model given in (2) is subject to a turbulent wind gust load. In this analysis, we assume that the wind gust model is described by

$$
\left\{\begin{array}{l}
\dot{x}_{w}=A_{w} x_{w}+B_{w} w_{g} \\
y_{w}=C_{w} x_{w}+D_{w} w_{g}
\end{array}\right.
$$

where $x_{w}$ denotes the states, $w_{g}$ the random gust wind which is assumed to be zero-mean white noise with intensity $W$, and $y_{w}$ the total random gust load applied to both rigid body aircraft and aeroelastic wings. We assume that $A_{w}$ is Hurwitz. If Dryden's longitudinal wind turbulence model is used, then $x_{w}$ will be consisting of vertical airspeed, vertical acceleration, and pitch rate. Adding the gust load $y_{w}$ to (2) to obtain

$$
\dot{x}_{p}=A_{p} x_{p}+B_{p} u_{\delta}+y_{w} .
$$




\section{II.B. Actuator Dynamics}

The actuator dynamics that produce control surface deflections $u_{\delta}$ are assumed to be derived from the following linear systems,

$$
\left\{\begin{array}{l}
\dot{x}_{\delta}=A_{\delta} x_{\delta}+B_{\delta} u \\
u_{\delta}=C_{\delta} x_{\delta}
\end{array}\right.
$$

where $x_{\delta}$ is the states of actuator dynamics and $u$ the control command. It is assumed that the pair $\left(A_{\delta}, B_{\delta}\right)$ is controllable and $A_{\delta}$ Hurwitz. For a 2nd order actuator, $x_{\delta}$ will be consisting of control surface deflection angle and angular rate. It is important to note that, in practice, $x_{\delta}$ is bounded.

\section{II.C. Open-loop System Representation}

After substituting (3) and (5) into (4), we obtain the overall open-loop state-space representation as follows,

$$
\dot{\eta}=A_{\eta} \eta+B_{\eta} u+D_{\eta} w_{g}
$$

where $\eta=\left(x_{p}, x_{w}, x_{\delta}\right)$ and

$$
A_{\eta}=\left[\begin{array}{ccc}
A_{p} & C_{w} & B_{p} C_{\delta} \\
0 & A_{w} & 0 \\
0 & 0 & A_{\delta}
\end{array}\right] ; B_{\eta}=\left[\begin{array}{c}
0 \\
0 \\
B_{\delta}
\end{array}\right] ; D_{\eta}=\left[\begin{array}{cc}
D_{w} & B_{p} \\
B_{w} & 0 \\
0 & 0
\end{array}\right] .
$$

It should be noted that the turbulence state $x_{w}$ is not controllable. In this paper, the control/performance outputs are denoted by $y$ and the sensor measured outputs by $z$, and they are described by

$$
\left\{\begin{array}{l}
y=C_{\eta} \eta \\
z=E_{\eta} \eta+v
\end{array}\right.
$$

where $C_{\eta}$ indicates the control outputs of interest and $E_{\eta}$ the locations of measurement sensors, and $v$ denotes sensor noise which is assumed to be zero mean white noise with intensity $V$. We can partition the performance output $y$ into a series of block outputs as

$$
y=\left[\begin{array}{c}
y_{1} \\
y_{2} \\
\vdots \\
y_{m}
\end{array}\right], y_{i}=\left(C_{\eta}\right)_{i} \eta .
$$

The reason for grouping control output $y$ in (8) is that for systems of large dimensions, the control objectives and constraints are often prescribed with respect to a collection of system states. For instance, we may choose $y_{1}=x_{\delta}$ to represent the constraints on actuator position and/or rate limits, and $\left(y_{2}, \cdots, y_{m}\right)$ the constraints on deflections and/or rates for aeroelastic wings.

Combining (6) and (7) yields the following full-order open-loop system description,

$$
\Sigma:\left\{\begin{aligned}
\dot{\eta} & =A_{\eta} \eta+B_{\eta} u+D_{\eta} w_{g} \\
y & =C_{\eta} \eta \\
z & =E_{\eta} \eta+v
\end{aligned}\right.
$$

Note that the dimension of $\eta$ can be very large. Henceforth, (9) or its equivalent form is called full-order evaluation model, while the design model, to be introduced later, represents the reduced-order model from which a stabilizing controller is designed.

\section{II.D. Control Design Objective}

The aircraft flight control system development cycle often requires optimization of control law subject to a chosen set of handling qualities specifications. ${ }^{7}$ In this study, we let $y_{\text {spec }}$ to denote such set, which captures 
aircraft rigid body performance, and let $\mathcal{S}_{\mathcal{I}}$ to denote the Level 1 requirements for $y_{\text {spec. For controlling }}$ the vibrational motion of aeroelastic wings, we let $\bar{Y}_{j}>0$ denote the desired output covariance constraint matrix for $y_{j}$, where $j=1,2, \cdots, m$. In this paper, the control design objective is to find a stable, strictly proper, dynamic output feedback controller of the form

$$
\left\{\begin{array}{rl}
\dot{x}_{c} & =A_{c} x_{c}+L_{c} z \\
u & =K_{c} x_{c}
\end{array}, x_{c} \in R^{n_{c}}\right.
$$

that stabilizes the full-order evaluation model $\Sigma$ described in Eq. (9), while minimizing the weighted control effort

$$
\mathcal{U}=\lim _{\tau \rightarrow \infty} \mathcal{E}\left\{u^{t}(\tau) R u(\tau)\right\}
$$

subject to

1. $y_{\text {spec }} \in \mathcal{S}_{\mathcal{I}}$ or all specifications are in Level 1 regions, and

2. output covariance matrix $Y_{j}$ for aeroelastic wings is constrained by $\bar{Y}_{j}$, i.e.

$$
\lim _{\tau \rightarrow \infty} \mathcal{E}\left\{y_{j}^{t}(\tau) y_{j}(\tau)\right\}=Y_{j} \leq \bar{Y}_{j}, j=1,2, \cdots, m
$$

Note that $L_{c}$ and $K_{c}$ are control gain matrices, $\mathcal{E}$ denotes an expectation operator, and $R$ is a sign definite symmetric weighting matrix. In general, the order of evaluation model can be very large, hence it is neither practical nor necessary to implement a full-order controller for (9). However, one may choose to design a full-order controller for initial assessment of reasonable or achievable output covariance constraint matrices of the closed-loop system, and use these to set $\bar{Y}_{j}$ for design iterations. In this paper, we will design a controller of the form (10) for a reduced-order design model.

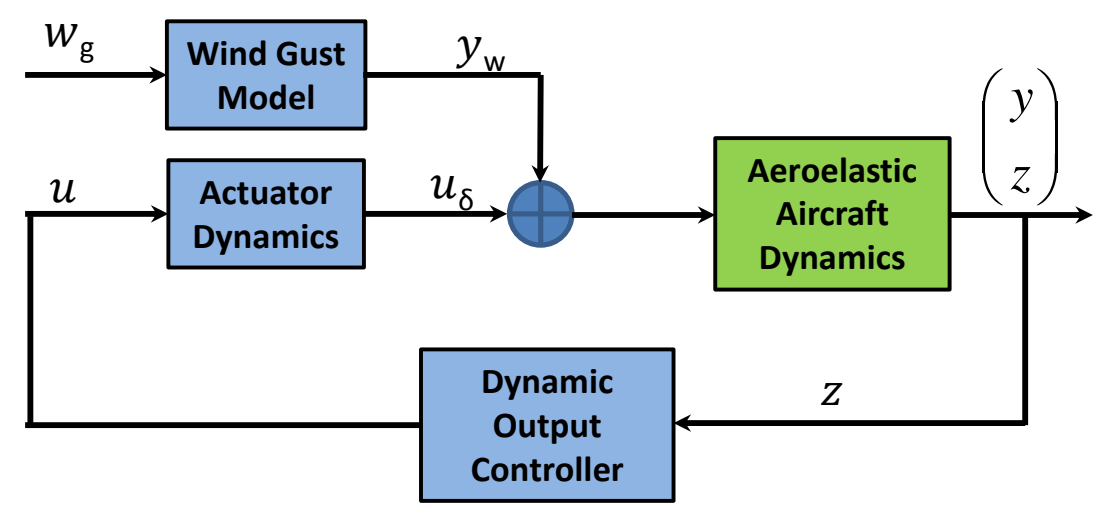

Figure 1. Closed-loop block diagram for aeroelastic aircraft model

\section{Modal Cost Analysis}

Beacuse of practical limitations on control bandwidth and to reduce computational burden in actual implementation, model reduction is often an essential part of controller design for physical systems of large dimension. This paper adopts the notion of modal cost analysis (MCA $)^{5}$ and utilizes it to attain a reducedorder design model. The concept of MCA was derived from the component cost analysis (CCA), which was first introduced in Ref. 4 and applied to the control of large space flexible structures. ${ }^{4}$ The idea of CCA is to examine the contribution, and hence significance, of each state component to the mission objectives in a control system. A metric of component contribution can be calculated in terms of output covariance, from 
which contribution of each component was studied and ranked from high to low. The approach was used to derive a reduced-order model by neglecting less significant components. The MCA is an extension of CCA to the standard matrix-second-order systems, where mass, damping, and stiffness matrices are sign definite and symmetric. A modal transformation can be applied so that in modal coordinate each mode is decoupled. In this case, contribution of each mode to output covariance is examined directly and a reduced-order model can be determined by retaining only dominating modes. Applications of MCA to model reduction and control system design was studied in Refs. 8 and 10. In this paper, because of the coupling between aerodynamics and aircraft dynamics, the total mass, damping, and stiffness matrices are neither sign definite nor symmetric, as indicated in (1). However, the MCA method can still be applied with minor modification.

Recall $\Sigma$ described in (9),

$$
\Sigma:\left\{\begin{aligned}
\dot{\eta} & =A_{\eta} \eta+B_{\eta} u+D_{\eta} w_{g} \\
y & =C_{\eta} \eta \\
z & =E_{\eta} \eta+v
\end{aligned}\right.
$$

It should be noted that the modal cost analysis is performed only to the aeroelastic modes. Since $A_{p}$ is Hurwitz and has distinctive eigenvalues, there exists a nonsingular transformation matrix $T$ such that, with $\eta=T \hat{\eta}, \Sigma$ can be transformed into a block decomposed form as

$$
\hat{\Sigma}:\left\{\begin{aligned}
\dot{\hat{\eta}} & =\hat{A} \hat{\eta}+\hat{B} u+\hat{D} w_{g} \\
y & =\hat{C} \hat{\eta} \\
z & =\hat{E} \hat{\eta}+v
\end{aligned}\right.
$$

where $\hat{\eta}$ is partitioned into

$$
\hat{\eta}=\left[\begin{array}{c}
\hat{\eta}_{1} \\
\hat{\eta}_{2} \\
\vdots \\
\hat{\eta}_{2 N} \\
\vdots
\end{array}\right],
$$

and $\hat{\eta}_{i} \in R^{2}$ represents the modal states for $i$ th aeroelastic mode; $i=1,2, \cdots, 2 N$. Accordingly, the system matrices can be partitioned as follows,

$$
\begin{gathered}
\hat{A}=T^{-1} A_{\eta} T=\operatorname{diag}\left\{J_{1}, J_{2}, \cdots, J_{2 N}, \cdots\right\} ; J_{i}=\left[\begin{array}{cc}
a_{i} & -b_{i} \\
b_{i} & a_{i}
\end{array}\right], \\
\hat{B}=T^{-1} B_{\eta}=\left[\begin{array}{c}
\hat{B}_{1} \\
\hat{B}_{2} \\
\vdots \\
\hat{B}_{2 N} \\
\vdots
\end{array}\right], \hat{C}=C_{\eta} T=\left[\begin{array}{lllll}
\hat{C}_{1} & \hat{C}_{2} & \cdots & \hat{C}_{2 N} & \cdots
\end{array}\right], \\
\hat{D}=T^{-1} D_{\eta}=\left[\begin{array}{c}
\hat{D}_{1} \\
\hat{D}_{2} \\
\vdots \\
\hat{D}_{2 N} \\
\vdots
\end{array}\right], \hat{E}=E_{\eta} T=\left[\begin{array}{lllll}
\hat{E}_{1} & \hat{E}_{2} & \cdots & \hat{E}_{2 N} & \cdots
\end{array}\right] .
\end{gathered}
$$

Note that the matrix $J_{i}$ denotes a real block diagonal form for a pair of complex eigenvalues $a_{i} \pm j b_{i}$. 


\section{III.A. Computing Modal Cost}

The MCA considered in this paper is to study modal contribution of each aeroelastic mode, denoted as $\nu_{i}$, to a weighted output covariance cost function $\mathcal{V}$ given by

$$
\mathcal{V}=\lim _{\tau \rightarrow \infty} \mathcal{E}\left\{y^{t}(\tau) Q y(\tau)\right\}=\sum_{i=1}^{2 N} \nu_{i}+\nu_{o},
$$

when subject to random input $w_{g}$. In (15), $Q$ is a block diagonal symmetric and non-negative matrix, and $\nu_{o}$ denotes modal cost contribution from other modes such as aircraft rigid body dynamics and actuator dynamics, etc. In what follows, we calculate the open-loop modal cost $\nu_{i} \cdot{ }^{4}$

Recall (13). Since $A_{\eta}$ is Hurwitz and the pair $\left(A_{\eta}, D_{\eta}\right)$ is stabilizable, the following Lyapunov equation

$$
P \hat{A}^{t}+\hat{A} P+\hat{D} W \hat{D}^{t}=0
$$

renders a unique positive semi-definite symmetric solution $P$, where we recall $W$ is the covariance of $w_{g}$. Next, the modal cost $\nu_{i}$ can be obtained by ${ }^{4}$

$$
\nu_{i}=\operatorname{tr}\left[\hat{C}^{t} Q \hat{C} P\right]_{i i}, i=1,2, \cdots, 2 N,
$$

where $\operatorname{tr}(X)$ denotes the matrix trace of $X$ and $\left[\hat{C}^{t} Q \hat{C} P\right]_{i i}$ is a $2 \times 2$ output covariance matrix corresponding to $i$ th aeroelastic mode. Now, given $\nu_{i}$ we then can rank them based on their modal cost as

$$
\left|\nu_{1}\right| \geq\left|\nu_{2}\right| \geq \cdots\left|\nu_{n}\right| \cdots \geq\left|\nu_{2 N}\right|,
$$

where $\nu_{1}$ being the most critical mode and $\nu_{2 N}$ least critical mode. Note that $\nu_{i}$ can be negative, which indicates that this particular mode is in fact helping to reduce the total cost $\mathcal{V}{ }^{4}$ however the total cost is non-negative. It should cause no confusion that in (18) $\nu_{i}$ means the $i$ th rank modal cost, not necessarily the modal cost of $i$ th aeroelastic mode. It is important to emphasize that the choice of weighting matrix $Q$ directly affects the modal cost.

Remark: When the open-loop system contains unstable mode(s), the proposed MCA model reduction process can still be applied with modification. Since the covariance cost for unstable modes is infinity, these modes should by default be retained. The MCA is then performed for stable modes, following the same process as presented.

\section{III.B. Reduced-order Design Model}

Based on (18) we may choose to keep the significant $n$ aeroelastic modes, where $n \ll 2 N$. Furthermore, $n$ is the number of modes that can be accommodated in the control system synthesis, given its bandwidth. If $n$ is chosen, then we may re-arrange the modal states $\hat{\eta}$ in (13) and decompose it into $\left(x_{r}, x_{t}\right)$, where $x_{r}$ and $x_{t}$ denote the retained and truncated modal states, respectively. Therefore, (13) can be rewritten as follows,

$$
\begin{aligned}
& {\left[\begin{array}{c}
\dot{x}_{r} \\
\dot{x}_{t}
\end{array}\right]=\left[\begin{array}{cc}
A_{r} & 0 \\
0 & A_{t}
\end{array}\right]\left[\begin{array}{l}
x_{r} \\
x_{t}
\end{array}\right]+\left[\begin{array}{l}
B_{r} \\
B_{t}
\end{array}\right] u+\left[\begin{array}{c}
D_{r} \\
D_{t}
\end{array}\right] w} \\
& y=\left[\begin{array}{ll}
C_{r} & C_{t}
\end{array}\right]\left[\begin{array}{l}
x_{r} \\
x_{t}
\end{array}\right] \\
& z=\left[\begin{array}{ll}
E_{r} & E_{t}
\end{array}\right]\left[\begin{array}{l}
x_{r} \\
x_{t}
\end{array}\right]+v
\end{aligned}
$$

Recall that the retained state $x_{r}$ consists of $n$ aeroelastic modes, aircraft rigid body dynamics, actuator dynamics, and turbulence model. Now, we can present the following reduced-order design model to which a stabilizing controller will be designed.

$$
\Sigma_{r}:\left\{\begin{array}{l}
\dot{x}_{r}=A_{r} x_{r}+B_{r} u+D_{r} w_{g} \\
y_{r}=C_{r} x_{r} \\
z_{r}=E_{r} x_{r}+v
\end{array}\right.
$$


In $\Sigma_{r}$ we have neglected the influence of observation spillovers induced by the truncated high-order aeroelastic modes. Since the modal cost contributions of these modes to the output covariance are less significant, it is expected that the reduced-order design model $\Sigma_{r}$ captures the salient feathers of the full-order model, and hence is used to build a dynamic output feedback controller. Ultimately, this controller will be evaluated with full-order evaluation model (9).

To summarize the open-loop model reduction process, we first need to perform coordinate transformation to (9) to block diagonalize the system matrices. Then, solve the Lyapunov equation (16) for $P$ and calculate the modal cost $\nu_{i}$, and finally rank $\nu_{i}$ and determine the number of aeroelastic modes to be retained.

\section{Integrated Flight Control and Output Covariance Constraint Problem}

Recall the dynamic output feedback controller given in (10),

$$
\left\{\begin{aligned}
\dot{x}_{c} & =A_{c} x_{c}+L_{c} z \\
u & =K_{c} x_{c}
\end{aligned}\right.
$$

Here, we choose $A_{c}=A_{r}-L_{c} E_{r}+B_{r} K_{c}$ and $x_{c} \in R^{n_{r}}$. Though the order of (10) is chosen to be the same as that of reduced-order design model, it utilizes the full measurement information, including the truncated modes. When interconnecting (19) with (10), we obatin the feedback-controlled full-order system representation as

$$
\left\{\begin{aligned}
\dot{\mathcal{X}} & =\mathcal{A X}+\mathcal{D} \mathcal{W} \\
\mathcal{Y} & =\mathcal{C X}
\end{aligned}\right.
$$

where

$$
\mathcal{X}=\left[\begin{array}{c}
x_{r}-x_{c} \\
x_{c} \\
x_{t}
\end{array}\right], \mathcal{Y}=\left[\begin{array}{c}
y \\
u
\end{array}\right], \mathcal{W}=\left[\begin{array}{c}
w_{g} \\
v
\end{array}\right]
$$

and

$$
\mathcal{A}=\left[\begin{array}{ccc}
A_{r}-L_{c} E_{r} & 0 & -L_{c} E_{t} \\
L_{c} E_{r} & A_{r}+B_{r} K_{c} & L_{c} E_{t} \\
0 & B_{t} K_{c} & A_{t}
\end{array}\right], \mathcal{D}=\left[\begin{array}{cc}
D_{r} & -L_{c} \\
0 & L_{c} \\
D_{t} & 0
\end{array}\right], \mathcal{C}=\left[\begin{array}{ccc}
C_{r} & C_{r} & C_{t} \\
0 & K_{c} & 0
\end{array}\right]
$$

Note that in $\mathcal{A}$ the upper left $2 \times 2$ block matrix is precisely the closed-loop matrix when closing (10) with the design model $\Sigma_{r}$, and the off-diagonal terms are due to observation and control spillovers of truncated modal states. Therefore, the design of control gains $K_{c}$ and $L_{c}$ is first performed for the design model $\Sigma_{r}$.

\section{IV.A. Output Covariance Control for Design Model}

In this section, we consider the following reduced-order closed-loop system representation which is extracted from (21),

$$
\left\{\begin{array}{l}
\dot{\mathcal{X}}_{r}=\mathcal{A}_{r} \mathcal{X}_{r}+\mathcal{D}_{r} \mathcal{W} \\
\mathcal{Y}_{r}=\mathcal{C}_{r} \mathcal{X}_{r}
\end{array}\right.
$$

where

$$
\mathcal{X}_{r}=\left[\begin{array}{c}
x_{r}-x_{c} \\
x_{c}
\end{array}\right], \mathcal{Y}_{r}=\left[\begin{array}{c}
y_{r} \\
u
\end{array}\right]
$$

and

$$
\mathcal{A}_{r}=\left[\begin{array}{cc}
A_{r}-L_{c} E_{r} & 0 \\
L_{c} E_{r} & A_{r}+B_{r} K_{c}
\end{array}\right], \mathcal{D}_{r}=\left[\begin{array}{cc}
D_{r} & -L_{c} \\
0 & L_{c}
\end{array}\right], \mathcal{C}_{r}=\left[\begin{array}{cc}
C_{r} & C_{r} \\
0 & K_{c}
\end{array}\right] .
$$

The output covariance constraint (OCC) problem $^{9}$ for $(22)$ is stated as follows:

For $\Sigma_{r}$, find a dynamic output feedback controller of the form

$$
\left\{\begin{aligned}
\dot{x}_{c} & =\left(A_{r}-L_{c} E_{r}+B_{r} K_{c}\right) x_{c}+L_{c} z_{r} \\
u & =K_{c} x_{c}
\end{aligned}\right.
$$


so that it minimizes the control cost

$$
\lim _{\tau \rightarrow \infty} \mathcal{E}\left\{u^{t}(\tau) R u(\tau)\right\} ; R>0,
$$

subject to the output covariance constraint matrices $\bar{Y}_{i}$, i.e.

$$
\left(C_{r}\right)_{i} P\left(C_{r}\right)_{i}^{t}=Y_{i} \leq \bar{Y}_{i} ; i=1,2, \cdots, m,
$$

where $P \geq 0$ solves the following Lyapunov equation

$$
A_{r} P+P A_{r}^{t}+D_{r} \hat{W} D_{r}^{t}=0,
$$

and $\hat{W}=\operatorname{diag}(W, V)$ denotes the covariance for $\mathcal{W}$.

The following lemma provides the solution to the OCC problem for (22).

Lemma $1 .{ }^{9}$ Consider the closed-loop system defined in (22). Suppose $\left(L_{c}, K_{c}\right)$ is the optimal solution to the OCC problem. Then, there exist a positive definite symmetric matrix $R$ and a block diagonal matrix $Q$ of the form

$$
Q=\operatorname{diag}\left\{Q_{1}, Q_{2}, \cdots, Q_{m}\right\} \geq 0,
$$

such that the control gain matrices $K_{c}$ and $L_{c}$ are given by

$$
K_{c}=-R^{-1} B_{r}^{t} X, L_{c}=Y E_{r}^{t} V^{-1},
$$

where $X \geq 0$ and $Y \geq 0$ are solutions to the control and filtering Riccati equations,

$$
\begin{aligned}
& 0=X A_{r}+A_{r}^{t} X-X B_{r} R^{-1} B_{r}^{t} X+C_{r}^{t} Q C_{r} \\
& 0=A_{r} Y+Y A_{r}^{t}-Y E_{r}^{t} V^{-1} E_{r} Y+D_{r} \hat{W} D_{r}^{t}
\end{aligned}
$$

Furthermore, the chosen weighting matrices and control gains satisfy the following output covariance constraints,

$$
\begin{aligned}
& 0=\left(Y_{i}-\bar{Y}_{i}\right) Q_{i} \\
& Y_{i}=\left(C_{r}\right)_{i}\left(Y+X_{c}\right)\left(C_{r}\right)_{i}^{t} ; i=1,2, \cdots, m,
\end{aligned}
$$

where $X_{c} \geq 0$ solves

$$
0=\left(A_{r}+B_{r} K_{c}\right) X_{c}+X_{c}\left(A_{r}+B_{r} K_{c}\right)^{t}+L_{c} V L_{c}^{t} .
$$

Therefore, if $K_{c}$ and $L_{c}$ are chosen according to (25), then both $\left(A_{r}+B_{r} K_{c}\right)$ and $\left(A_{r}-L_{c} E_{r}\right)$ are Hurwitz, so are $\mathcal{A}_{r}$ and $\mathcal{A}$, since $A_{t}$ is Hurwitz. In Ref. 9, an algorithm for selecting weighting matrices was presented and an optimal solution to the OCC control problem obtained, as stated in the Lemma. It is important to note that the OCC problem described above is for the reduced-order design model. We will incorporate this process into the OCC control problem for full-order evaluation model when we develop an integrated modeling and control approach in Section V.

\section{IV.B. Optimal Flight Control Design in CONDUIT}

CONDUIT (CONtrol Designer's Unified InTerface) is a state-of-the-art multidisciplinary computational facility for aircraft flight control design, evaluation, and integration. It incorporates aircraft dynamic models, handling qualities evaluation, closed-loop system performance, and multi-objective optimization into a MATLAB/SIMULINK based interactive environment. For detailed description of CONDUIT and its applications to flight control development projects, one may refer to Refs. 11-14. Here we provide only a brief overview of CONDUIT.

To setup a problem in CONDUIT, we first need to define the aircraft dynamics, actuator dynamics, and architecture of the control law in SIMULINK. Then, select a set of appropriate design specifications for performance evaluation from the provided specification libraries, or create user-defined specifications using CONDUIT's SpecMaker tool. In each design specification there are three levels of compliance, namely, Level 1, Level 2, and Level 3. ${ }^{12}$ A sample design specification for stability gain/phase margin (StbMgG1) is shown in Figure 2, where Level 1 region, colored in blue, is the most desirable performance region indicating gain 


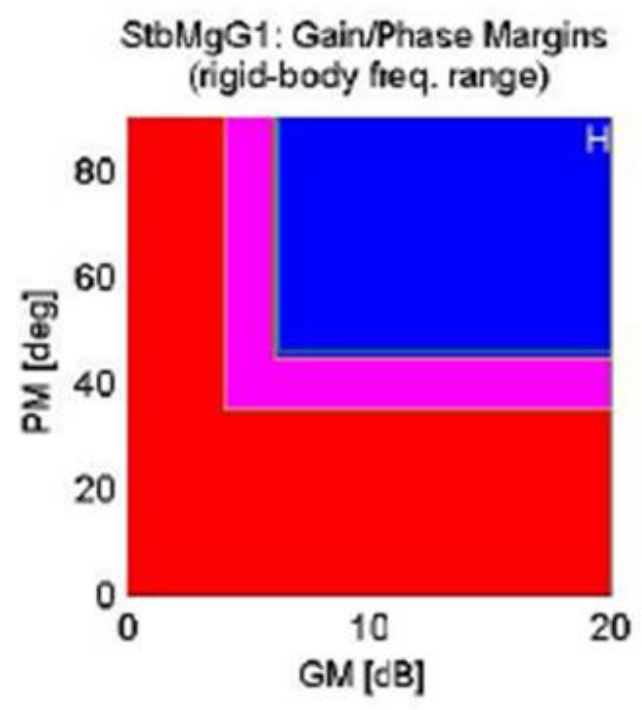

Figure 2. Sample Design Specification: Stability Gain/Phase Margins

margin above $6 \mathrm{~dB}$ and phase margin above $45^{\circ}$. Level 2, colored in magenta, means the performance is adequate, and Level 3, colored in red, indicates an inadequate performance region. Note that Level 1/2/3 boundaries can be adjusted to suit user's specifications.

Next, prioritize each design specification by designating it as hard constraint, soft constraint, or summedobjective. ${ }^{11}$ Finally, CONDUIT utilizes CONSOL-OPTCAD as optimization engine and conducts multiobjective optimization in three distinct phases. In Phase 1, the control law is tuned so that all hard constraints reach Level 1 region. Phase 2 will work on the soft constraints and ensure they reside in Level 1 region, whereas in Phase 3, CONDUIT will optimize the control law to the selected summed-objective performance criteria, therefore to attain the best control design from the family of feasible solutions.

In this paper, the design specifications include not only for aircraft rigid body dynamics, but for aeroelastic wings. The vibrational behavior of the wings, which is captured in $y_{j}$, will be constrained as output covariance $\bar{Y}_{j}$ and needs to be incorporated in CONDUIT as part of handling qualities specifications. Therefore, we need to create a set of OCC specifications for full-order evaluation model (9). In what follows, we assume $y_{j}$ be a scalar output taken at some location along the flexible wing, and $\bar{Y}_{j}$ a desired output covariance. Let $\hat{Y}_{j}>\bar{Y}_{j}$ be an acceptable output covariance for $y_{j}$. Then, using SpecMaker tool in CONDUIT, we can create an OCC design specification, AseOcG1, shown in Figure 3.

Therefore, in CONDUIT the complete handling qualities and performance specifications will consist of those for rigid body dynamics, $y_{\mathrm{spec}}$, and aeroelasticity. CONDUIT optimization process will then tune the weighting matrices, and hence the control gains $\left(K_{c}, L_{c}\right)$, until $y_{\text {spec }} \in \mathcal{S}_{\mathcal{I}}$ and $Y_{j} \leq \bar{Y}_{j}$.

\section{Iterative Modeling and Control Design Process}

We present in this section an iterative algorithm that involves model reduction, OCC controller design, and optimal flight control design, subject to aircraft handling qualities and performance specifications. The design process searches for the controller with the best performance by tuning the design model until the iteration converges, as illustrated in Figure 4. The model reduction utilizes MCA, and the design of flight controller for rigid body dynamics and aeroelastic wings follows the procedure presented earlier. This is essentially a closed-loop model reduction process, since the choice of weighting matrix $Q$ directly affects the modal cost, and hence model reduction process. On the other hand, matrix $Q$ also affects the computation for output covariance, in that if a particular output covariance specification $\bar{Y}_{j}$ is not satisfied then the 


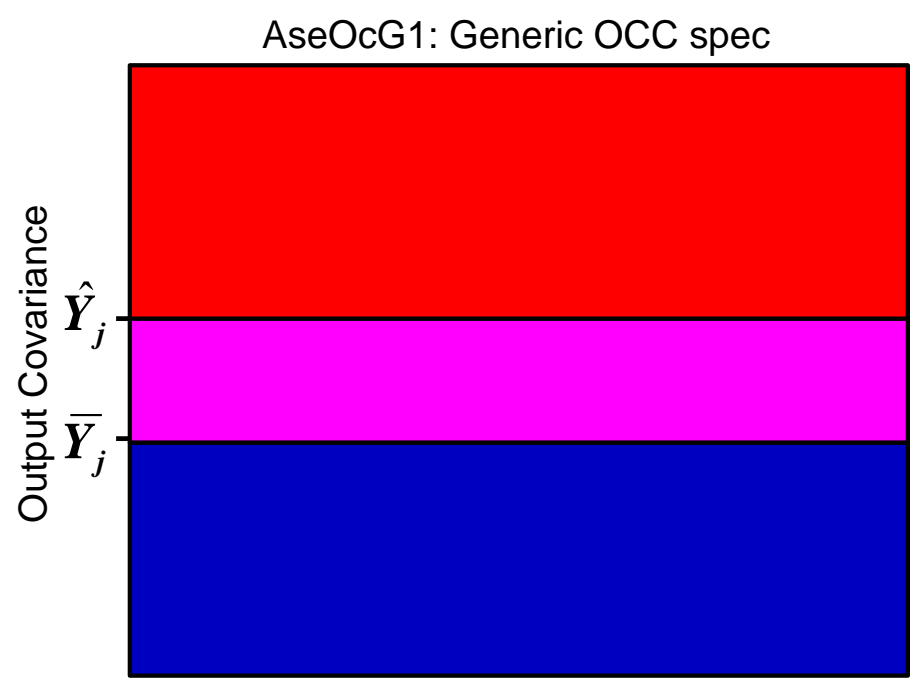

Figure 3. OCC Design Specification

corresponding $Q_{j}$ will be adjusted for next iteration. Therefore, by combining model reduction and OCC control problem, we are able to find an appropriate weighting matrix $Q$ that reflects the importance of each output and that the reduced-order design model retains the dominant dynamic characteristics which is important to attain the required flight control performance.

CONDUIT's external scripting capability ${ }^{14}$ is quite suited for performing the iterative control design process depicted in Figure 4. The external scripting file is a MATLAB m-file which allows us to incorporate the OCC control problem in CONDUIT and executed with optimization process.

\section{Applications to Generic Transport Model}

Here, we present an application of proposed integrated OCC algorithm to longitudinal aeroelastic GTM as an illustraive example. GTM is configured with 6 trailing edge flaps and 6 leading edge slats, in addition to the elevator, see Figure 5. The objective is to design an optimal reduced-order output feedback controller for "full-order"' GTM, so that the closed-loop system satisfies the desired handling qualities/performance requirements, and the vibartional motion of the wings is suppressed.

In this paper, GTM at cruise condition at Mach 0.8 and altitude at 30,000 ft with $50 \%$ fuel remaining is considered. Furthermore, we let $N=10$, that is, we account for 10 bending and 10 torsional modes. It is assumed that both longitudinal rigid body states and aeroelastic wing states are available for measurement. For aeroelastic measurements, we take the outputs at 10 equally spaced data points along the wing span; and the last point being at the wing tip. We measure both displacement and rate at these 10 points. The 5 control outputs for aeroelastic wings are assumed to coincide with the last 5 measurement points. For this analysis, we assume that GTM is subject to a random gust turbulence of $\pm 20 \mathrm{ft} / \mathrm{s}$ and random measurement noise of $0.001 \mathrm{ft}{ }^{2}\left(\mathrm{rad}^{2}\right)$.

\section{VI.A. Open-loop Analysis for GTM}

We choose $Q$ to be an identity matrix and follow the open-loop MCA procedure described in Section III. Table 1 contains the list of modal costs, along with their modal frequency and damping.

The open-loop MCA shown in Table 1 indicates that beyond 4th bending and 2nd torsional mode the modal cost becomes less significant. Higher mode demands larger control bandwidth, therefore, given the frequency range of actuator dynamics and results of $\mathrm{MCA}$, we can decide specific aeroelastic modes to retain in the 


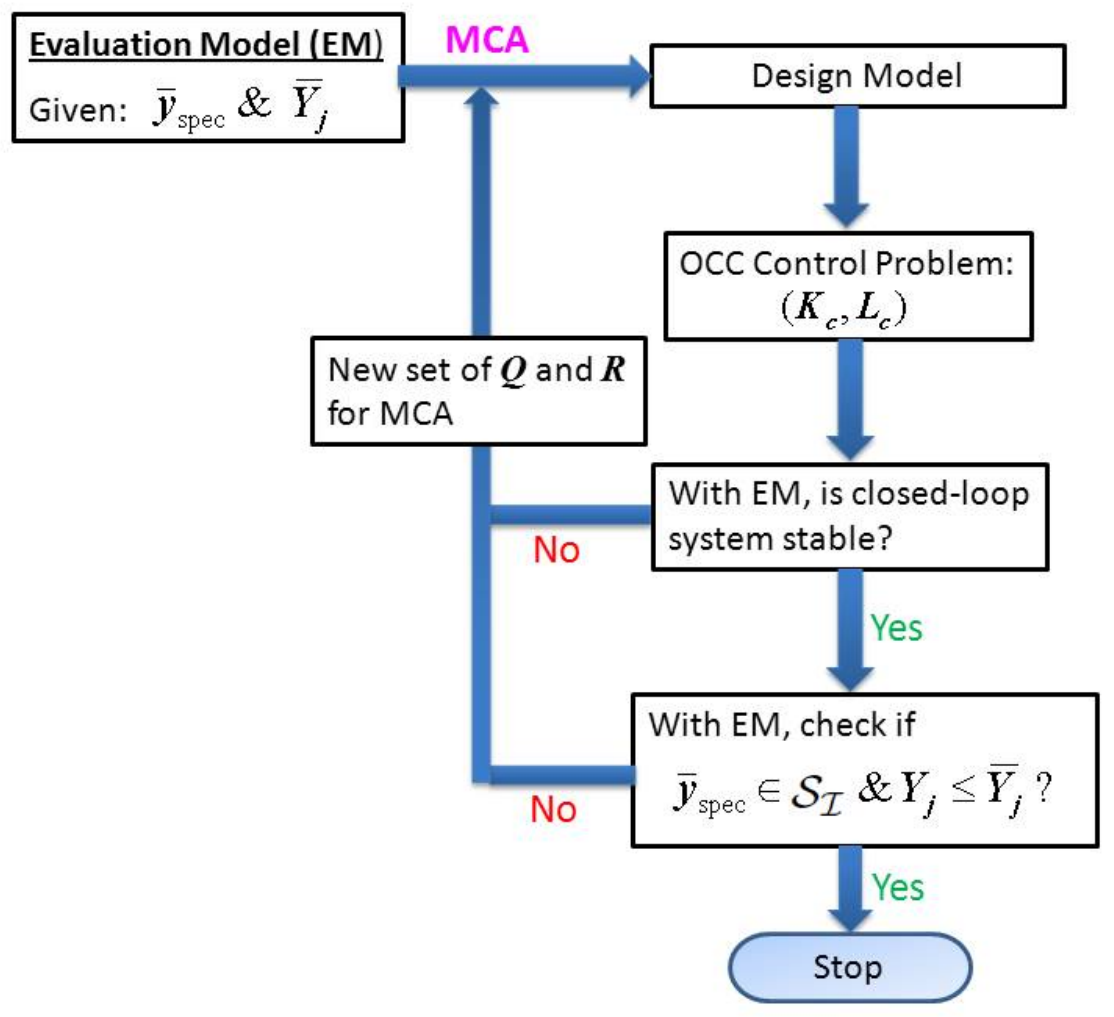

Figure 4. Integrated Modeling and Control Algorithm

Table 1. Open-loop Modal Cost Analysis for GTM

\begin{tabular}{|c|c|c|c|}
\hline Mode ID & Modal Cost $\left(10^{6}\right)$ & Frequency (rad/sec) & Damping \\
\hline \hline 1st torsion & 0.281 & 8.47 & 0.011 \\
\hline 1st bending & 0.017 & 8.71 & 0.289 \\
\hline 2nd bending & 0.039 & 14.52 & 0.210 \\
\hline 2nd torsion & 1.326 & 15.70 & 0.007 \\
\hline 3rd bending & 0.0027 & 24.49 & 0.077 \\
\hline 4th bending & 0.0024 & 31.86 & 0.067 \\
\hline$\vdots$ & $\vdots$ & $\vdots$ & $\vdots$ \\
\hline
\end{tabular}




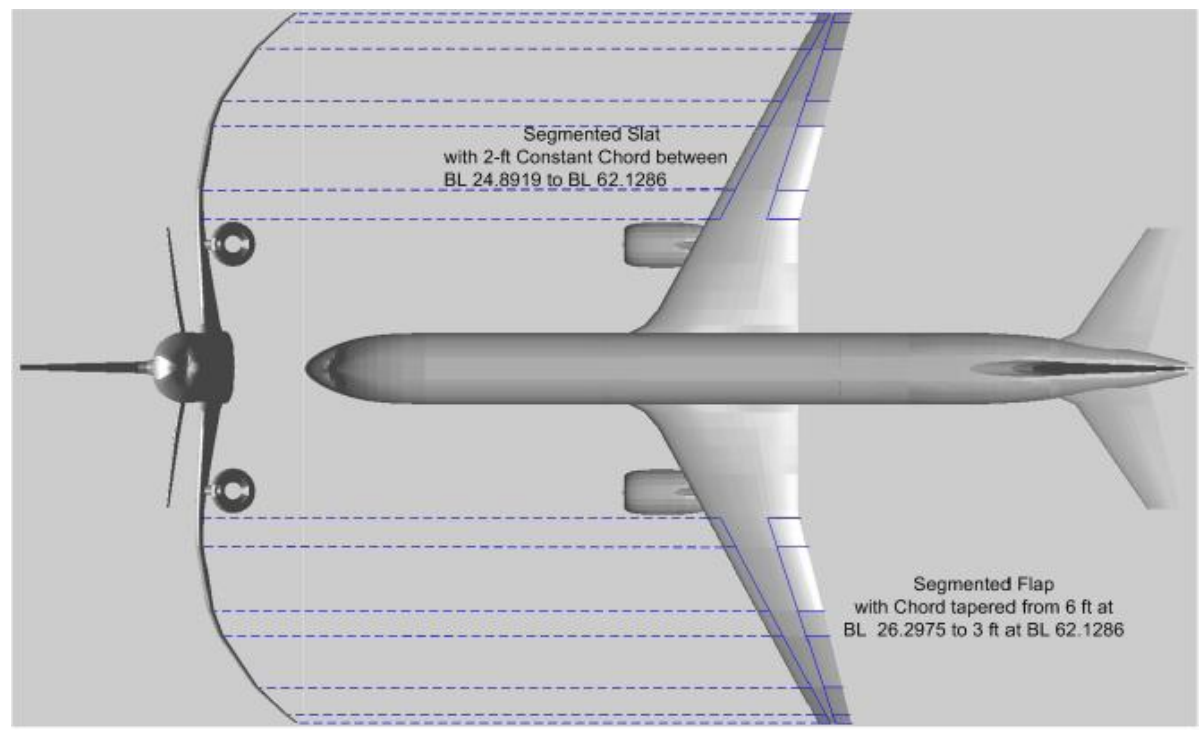

(Re-print from IPP Report by N. Nguyen)

Figure 5. Generic Transport Model 
design model. We may start with retaining 6 modes; namely, 1 st to 4 th bending mode and 1 st to 2 nd torsional mode, and design a reduced-order dynamic output feedback controller given in (10) by following the procedure presented in Section IV.A.

Before proceeding, we need to access the level of output covariance of the open-loop system. This will help us gauge and setup the achievable output covariance level for closed-loop system for optimization process. The output covariances for open-loop system are shown in Table 2.

Table 2. Open-loop Output Covariance

\begin{tabular}{|c|c|c|}
\hline Data Point & Bending Deflection $\times 10^{-2}\left(\mathrm{ft}^{2}\right)$ & Torsional Deflection $\times 10^{-6}\left(\mathrm{rad}^{2}\right)$ \\
\hline \hline Pt. 1 & 0.52 & 1.71 \\
\hline Pt. 2 & 1.03 & 2.79 \\
\hline Pt. 3 & 1.71 & 4.07 \\
\hline Pt. 4 & 2.52 & 5.38 \\
\hline Pt. 5 & 4.17 & 6.79 \\
\hline
\end{tabular}

\section{VI.B. Probelm Setup in CONDUIT}

The emphasis in this section is to define an iterative control optimization problem to be solved in CONDUIT. We have chosen six design specifications for analysis; they are: Eignevalues, Stability Gain/Phase Margins, Quickness, Crossover Frequency, Actuator Saturation, and Normalized Output Covariance. The eigenvalues specification verifies the closed-loop system is stable, the stability margin specification ensures that the satisfactory gain and phase margins are attained for the broken-loop response, the crossover frequency specification is to ensure minimal overdesign, pitch axis quickness low bandwidth specification is to ensure the ratio of peak attitude rate to change in attitude be exceeding specified limits, the actuator saturation specification is used to limit the actuator saturation in position and rate over a specified period, and the output covariance specification is to ensure the vibrational motion of the wings is contained. These specifications are listed in Table 3, along with their constraint type and level I specifications.

Table 3. Design Specifications Used in CONDUIT

\begin{tabular}{|c|c|c|c|c|}
\hline Requirement & Specification & Source & Constraint Type & Level I \\
\hline \hline Eigenvalues & EigLcG1 & Ames Research Center & Hard & $\operatorname{Re}\left(\lambda_{i}\right)<0$ \\
\hline Stability Margins & StbMgG1 & MIL-F-9490D & Hard & at least $6 d B / 45^{\circ}$ \\
\hline Quickness & QikAtG1 & Ames Research Center & Soft & As shown \\
\hline Crossover Frequency & CrsLnG1 & Ames Research Center & Summed Objective & $\leq 2 \mathrm{rad} / \mathrm{s}$ \\
\hline Actuator Saturation & SatAcG1 & Ames Research Center & Soft & $1 \mathrm{sec}$ \\
\hline Output Covariance & AseOcG1 & Ames Research Center & Hard/Soft & $\leq 10 \% \times \mathrm{OC}_{\text {open }}$ \\
\hline
\end{tabular}

Figure 6 shows the handling qualities (HQs) window in CONDUIT. It should be emphasized that the objective of OCC problem for aeroelastic wings is to achieve an order of magnitude less than the output covariance of the open-loop case, as indicated in Table 3. In this regard, Level I/Level II boundary of AseOcG1 is normalized to 1 . Note that there is a built in scale factor within specification that we could use to re-scale the level I region, if needed.

The tuning design parameters are contained in the 'Q' matrix. Let dpp_sp to indicate the tunable weighting parameter for longitudinal short period mode, dpp_bend for bending deflections, and dpp_tors for torsional deflections. It should be noted that, since the aircraft's rigid body dynamics is coupled with aeroelstic modes, and in addition the bending and torsional motions are coupled, the three weighting parameters can not be tuned independently. 


\section{VI.C. CONDUIT Optimization}

In this section, an optimal dynamic output feedback controller is designed for the reduced-order design model, which consists of rigid body short period mode and 6 aeroelastic modes listed in Table 1 . The controller is developed by solving the two Riccati equations given in Lemma 1 with varying weighting matrix $Q$. Subsequently, this reduced-order controller is applied to the full-order system $\Sigma$ in (9). The iterative optimal selection of weighting matrix is performed in CONDUIT subject to the list of design specifications given in Table 3. Figure 6 shows the evaluation of HQs with initial seletion of weighting parameters, i.e. dpp_sp $=$ dpp_bend $=$ dpp_tors $=0.0001$. These parameters reflect a small control effort by closing the feedback loop for initial check up. The results show that the closed-loop system is stable and satisfies overall performance requirements, but the output covariances in bending and torsional displacements fail to meet level I requirement. Figure 7 shows the HQs of optimal solutions after 19 iterations. It shows that all design specifications reach level I regions, and the optimal weighting parameters are dpp_sp $=0.2127$, dpp_bend $=0.0021$, dpp_tors $=17.6356$. Figures 8 and 9 show the comparisons between open-loop and closed-loop bending and torsional deflections at Pt.1 and Pt.5, respectively, subject to random gust turbulence.

We have also designed optimal output feedback controllers for varying number of retaining aeroelastic modes; namely, 5 modes (B1,B2,B3,T1,T2), 4 modes (B1,B2,T1,T2), and 3 modes (B1,B2,T2). In all these cases, the closed-loop full order system is stable and satisfies relevant performance requirements, except the output covariances, especially, the torsional deflections at outer wing (Pt.5). Figures 10 and 11 show the closed-loop output covariance comparisons with various design models. As expected, the closed-loop performance degrades as the order of controller reduces.

\section{VI.D. Flutter Analysis}

Figure 12 shows the root locus of the open-loop aeroelastic model as aircraft speed varies from Mach 0.6 to 0.9 , and at around Mach 0.82 the system becomes unstable (B1,T1). The designed optimal reducedorder controller is used for flutter analysis. Figure 13 shows the root locus of the closed-loop system with varying air speed from Mach 0.8 to 0.92, and Figures 14 and 15 show the output covariance performance comparisons. The closed-loop system is the interconnection of the open-loop aeroelastic model with the proposed reduced-order dynamic output feedback controller based on 6 aeroelastic modes. Note that the proposed dynamic controller is designed at Mach 0.8, the results in these figures indicate that the designed controller is fairly robust against modeling uncertainties, though the performance degrades somewhat. As expected, the closed-loop system stability breaks down when air speed continues to increase, in this case, beyond Mach 0.88 .

\section{Conclusion}

In this paper, we have presented an integrated model reduction and control system design process for aircraft with flexible wings. Model reduction is conducted using modal cost analysis for each aeroelastic mode, and the modes that show significant contribution to the output covariance are retained. Since the notion of output covariance control is new to the aircraft aeroelastic study, in this paper we have used $10 \%$ of the openloop output covariances as the closed-loop design requirement for aeroelastic modes. The optimal dynamic output feedback controller is designed for the reduced-order design model, by following a procedure similiar to $L_{2} / H_{\infty}$ multiobjective control design process. The process is to find the proper weighting parameters iteratively until the design objectives are met. The output covariance control algorithm is implemented in CONDUIT for full-order evaluation model. The analysis shows that the proposed controller is able to achieve level I design specifications for both rigid-body aircraft performance and flexible wings. It is also shown that the proposed controller is robust in the presence of modeling uncertainties.

\section{Acknowledgments}

The authors like to thank the CONDUIT support team at NASA Ames for their technical assistance. 


\section{References}

${ }^{1}$ Juang, J. N., Pappa, R. S., "'An Eigensystem Realization Algorithm for Modal Parameter Identification and Model Reduction,"' AIAA Journal of Guidance, Vol. 8, No. 5, 1984, pp. 620-627.

${ }^{2}$ Enns, D. F., "'Model Reduction with Balanced Realization: An Error Bound and a Frequency Weighted Generalization,"' Proc. of IEEE Conf. on Decision and Control, Las Vegas, NV., 1984, pp. 127-132.

${ }^{3}$ Glover, K., "'All Optimal Hankel-norm Approximations of Linear Multivariable Systems in Their $L^{\infty}$-Error Bounds,", Int. J. Control, Vol. 39, No. 6, 1984, pp. 1115-1193.

${ }^{4}$ Skelton, R. E., and Yousuff, A., "Component Cost Analysis of Large Scale Systems,"' Int. J. Control, Vol. 37, No. 2, 1983, pp. 285-304.

${ }^{5}$ Skelton, R. E., Hughes, P. C., and Hablani, H. B., " Order Reduction for Models of Space Structures Using Modal Cost Analysis,"' AIAA J. Guidance, Control, and Dynamics, Vol. 5, No. 4, 1982, pp. 351-357.

${ }^{6}$ Nguyen, N., Tuzcu, I., Yucelen, T., and Calise, A., "'Longitudinal Dynamics and Adaptive Control Application for an Aeroelastic Generic Transport Model,"' AIAA Atmospheric Flight Mechanics Conf., AIAA Paper 2011-6291, Portland, Or., 2011.

${ }^{7}$ United States Department of Defense, "'Flying Qualities of Piloted Vehicles,"' MIL-STD-1797, 1987.

${ }^{8} \mathrm{Zhu}$, G., and Skelton, R. E., "'Integrated Modeling and Control for the Large Spacecraft Control Laboratory Experiment Facility,"' AIAA J. Guidance, Control, and Dynamics, Vol. 17, No. 3, 1994, pp. 442-450.

${ }^{9}$ Zhu, G., Rotea, M. A., and Skelton, R. E., "'A Convergent Algorithm for the Output Covariance Constraint Control Problem,"' SIAM J. Control Optim., Vol. 35, No. 1, 1997, pp. 341-361.

${ }^{10} \mathrm{Zhu}$, G., Grigoriadis, K. M., and Skelton, R. E., "'Covariance Control Design for Hubble Space Telescope,"' AIAA J. Guidance, Control, and Dynamics, Vol. 18, No. 2, 1995, pp. 230-236.

${ }^{11}$ Tischler, M. B., Morel, M. R., Colbourne, J. D., Biezad, D. J., Levine, W. S., and Moldoveanu, V., "CONDUIT - A New Multidisciplinary Integration Environment for Flight Control Development,"' AIAA Paper 97-3773, 1997.

${ }^{12}$ Colbourne, J. D., Frost, C. R., Tischler, M. B., Cheung, K. K., Hiranaka, D. K., and Biezad, D. J., " Control Law Design and Optimization for Rotorcraft Handling Qualities Criteria Using CONDUIT," American Helicopter Society 55th Annual Forum, Montreal, Quebec, Canada, 1999.

${ }^{13}$ Tischler, M. B., Blanken, C. L., Cheung, K. K., Swei, S., Sahasrabudhe, V., and Faynberg, A., "'Modernized Control Laws for UH-60 Blackhawk Optimization and Flight-Test Results,"' AIAA J. Guidance, Control, and Dynamics, Vol. 28, No. 5, 2005, pp. 964-978.

${ }^{14}$ Tischler, M. B., Lee, J. A., and Colbourne, J. D., "'Comparison of Flight Control System Design Methods Using the CONDUIT Design Tool,"' AIAA J. Guidance, Control, and Dynamics, Vol. 25, No. 3, 2002, pp. 482-493. 


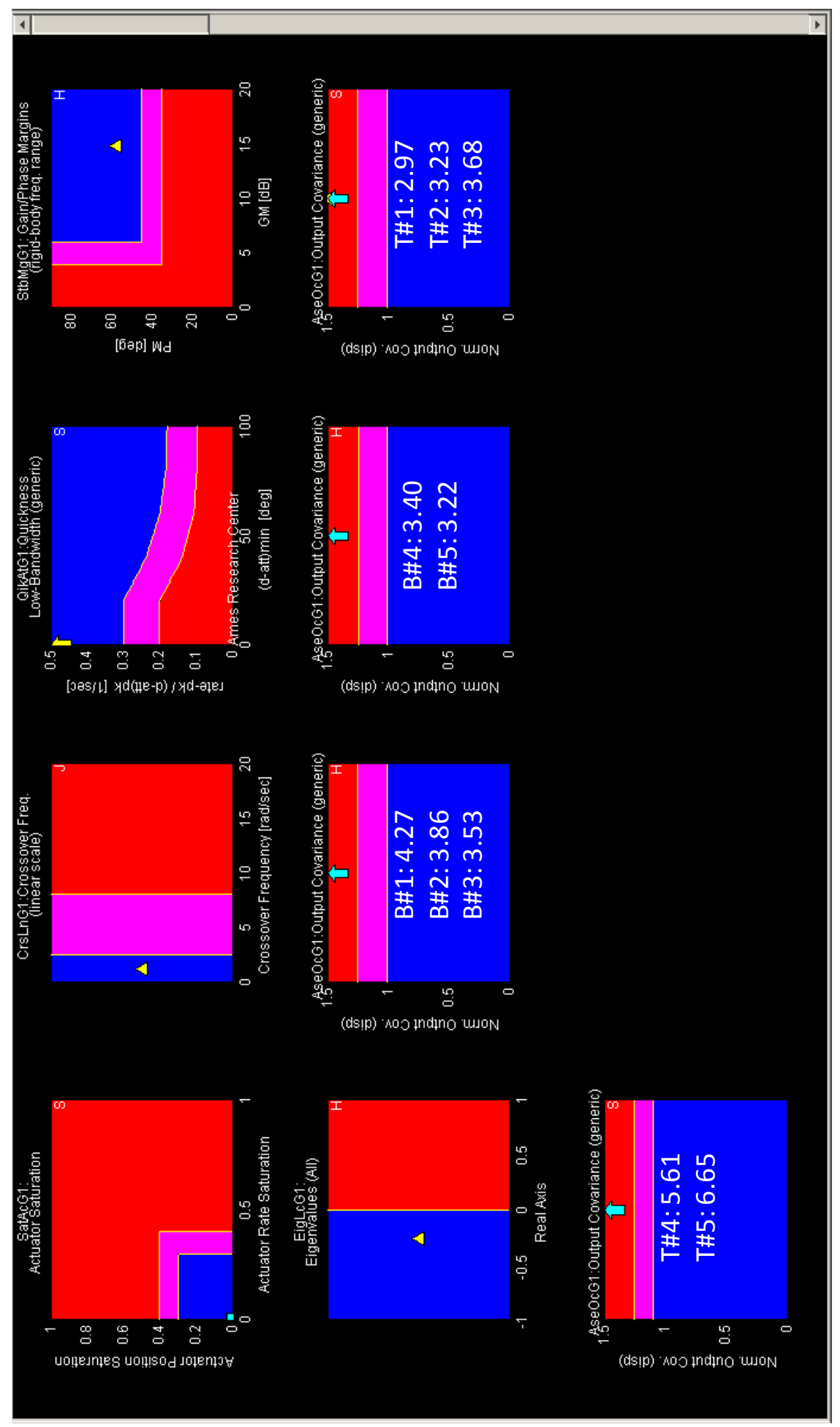

Figure 6. Handling Qualities at baseline gains 


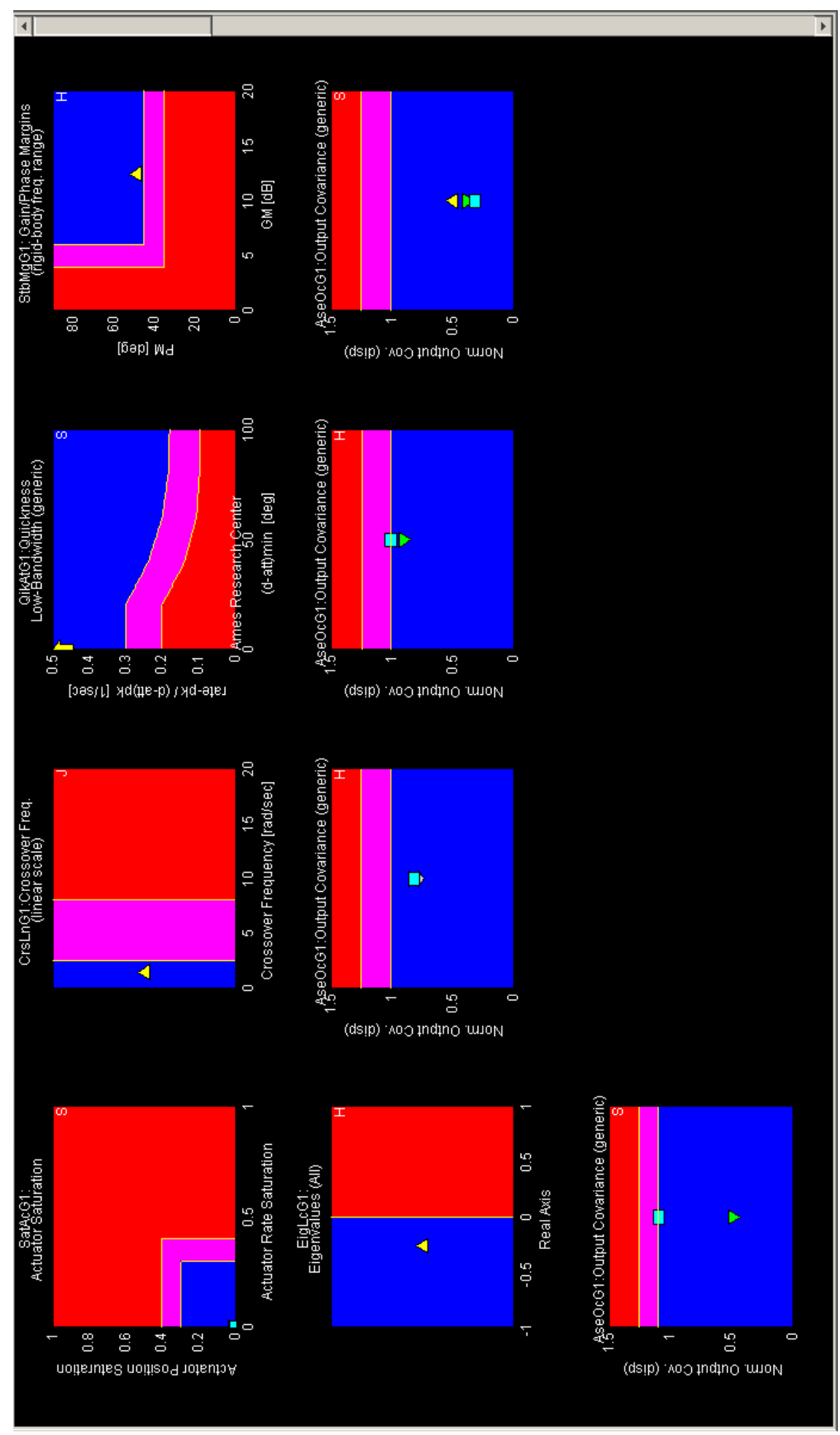

Figure 7. Handling Qualities at optimal gains 

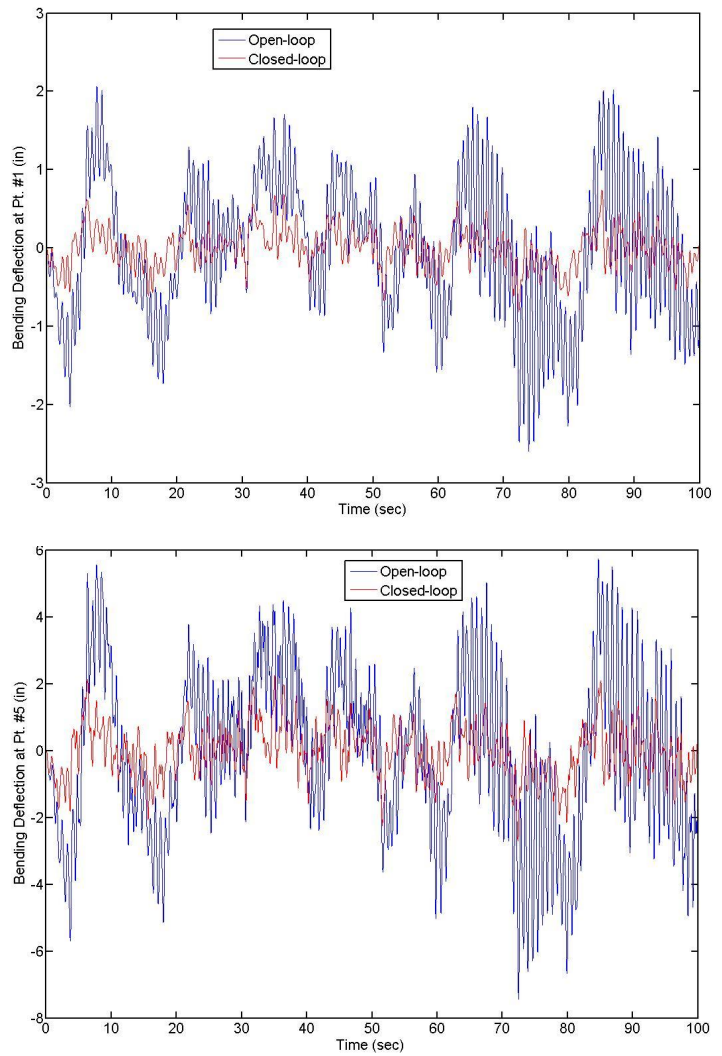

Figure 8. Bending displacement comparison between open and closed-loop at Pt. 1 and Pt. 5 

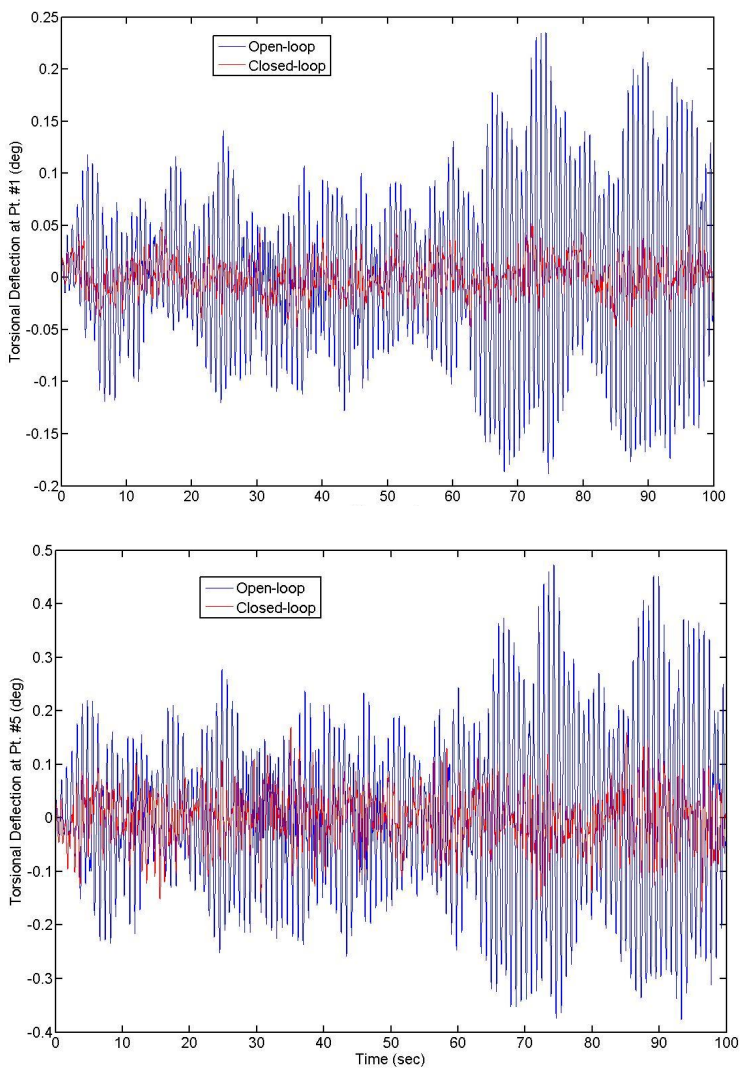

Figure 9. Torsional displacement comparison between open and closed-loop at Pt. 1 and Pt. 5 


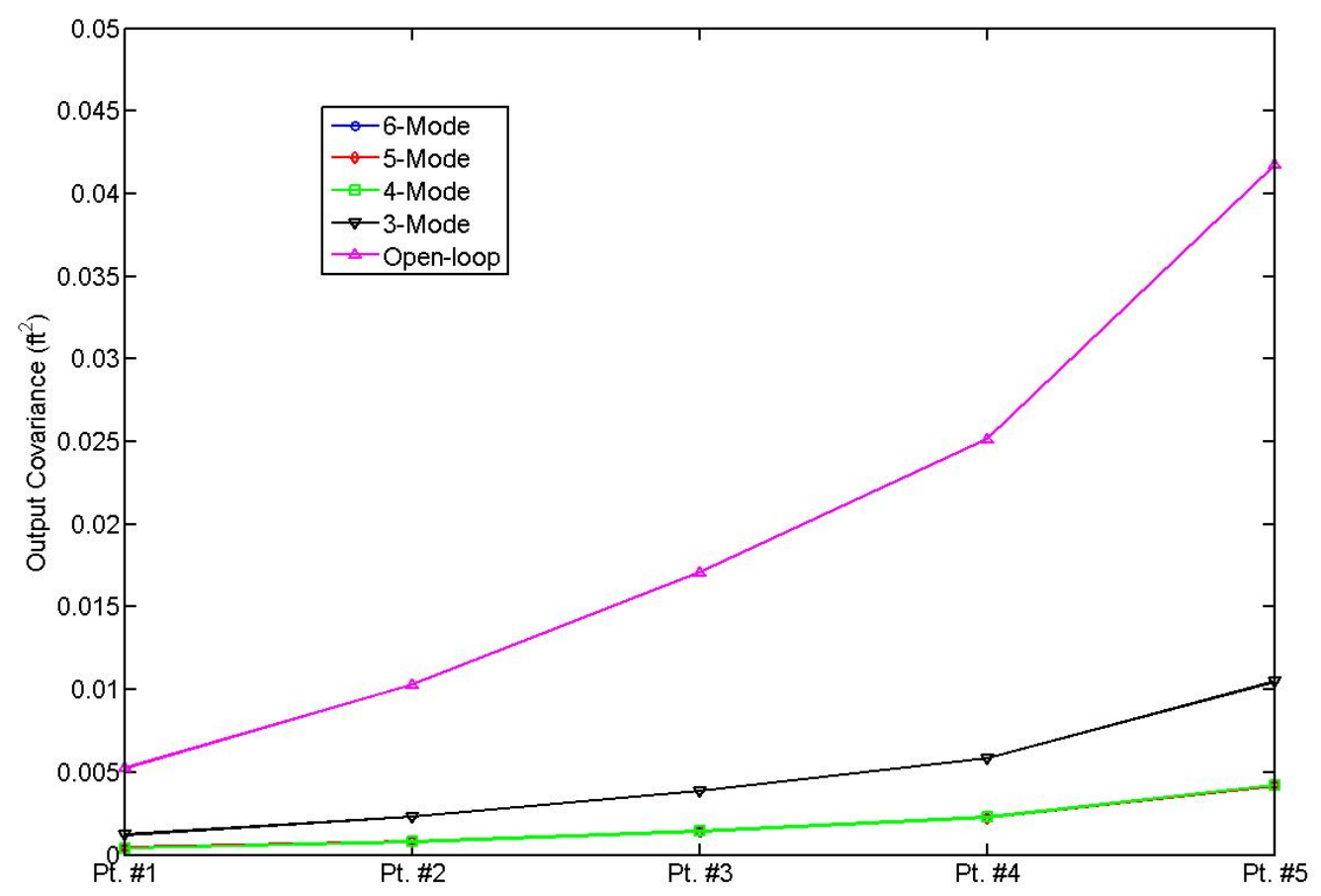

Figure 10. Output covariance (bending) comparison for 6-mode, 5-mode, 4-mode, and 3-mode

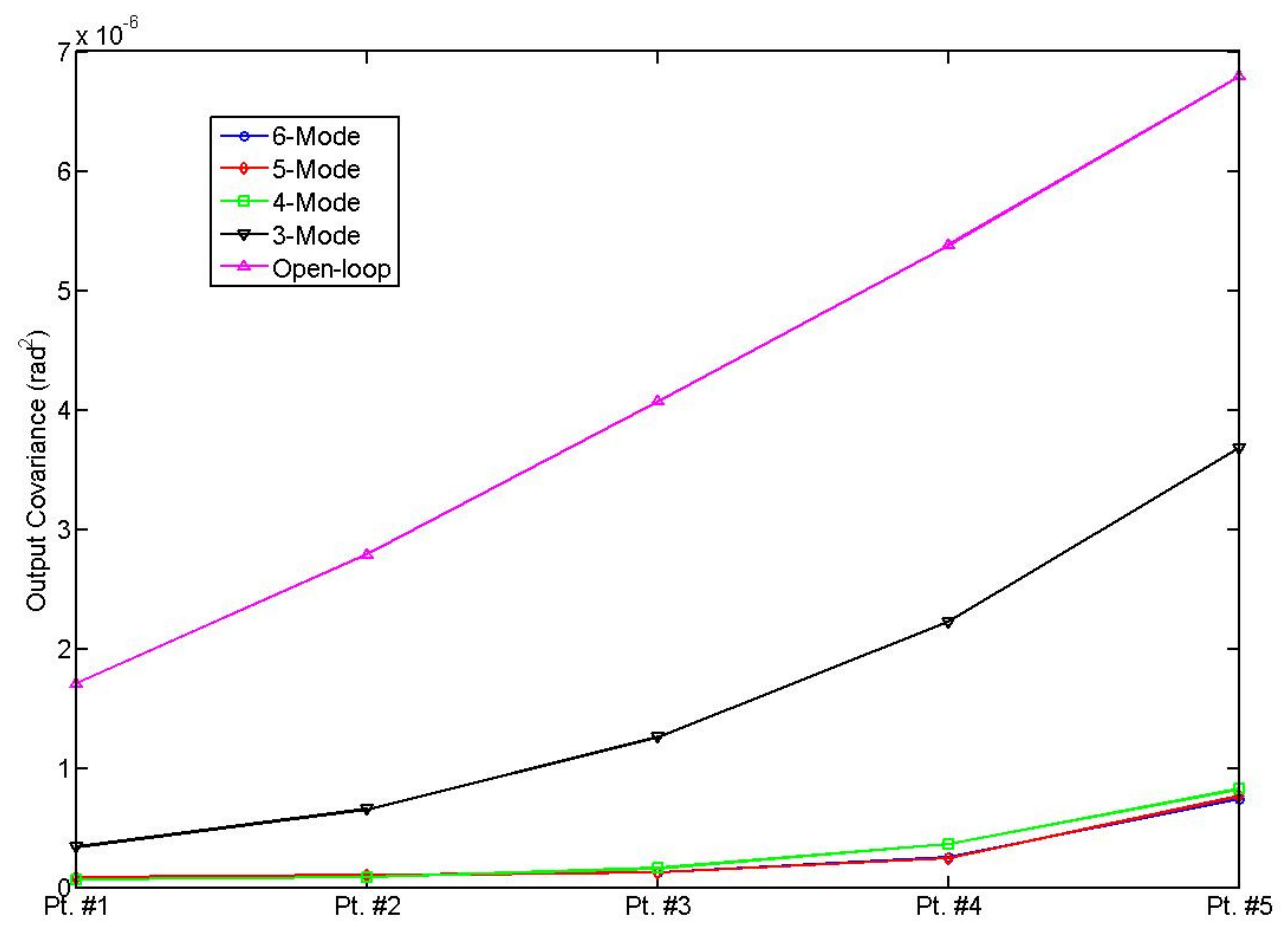

Figure 11. Output covariance (torsion) comparison for 6-mode, 5-mode, 4-mode, and 3-mode 


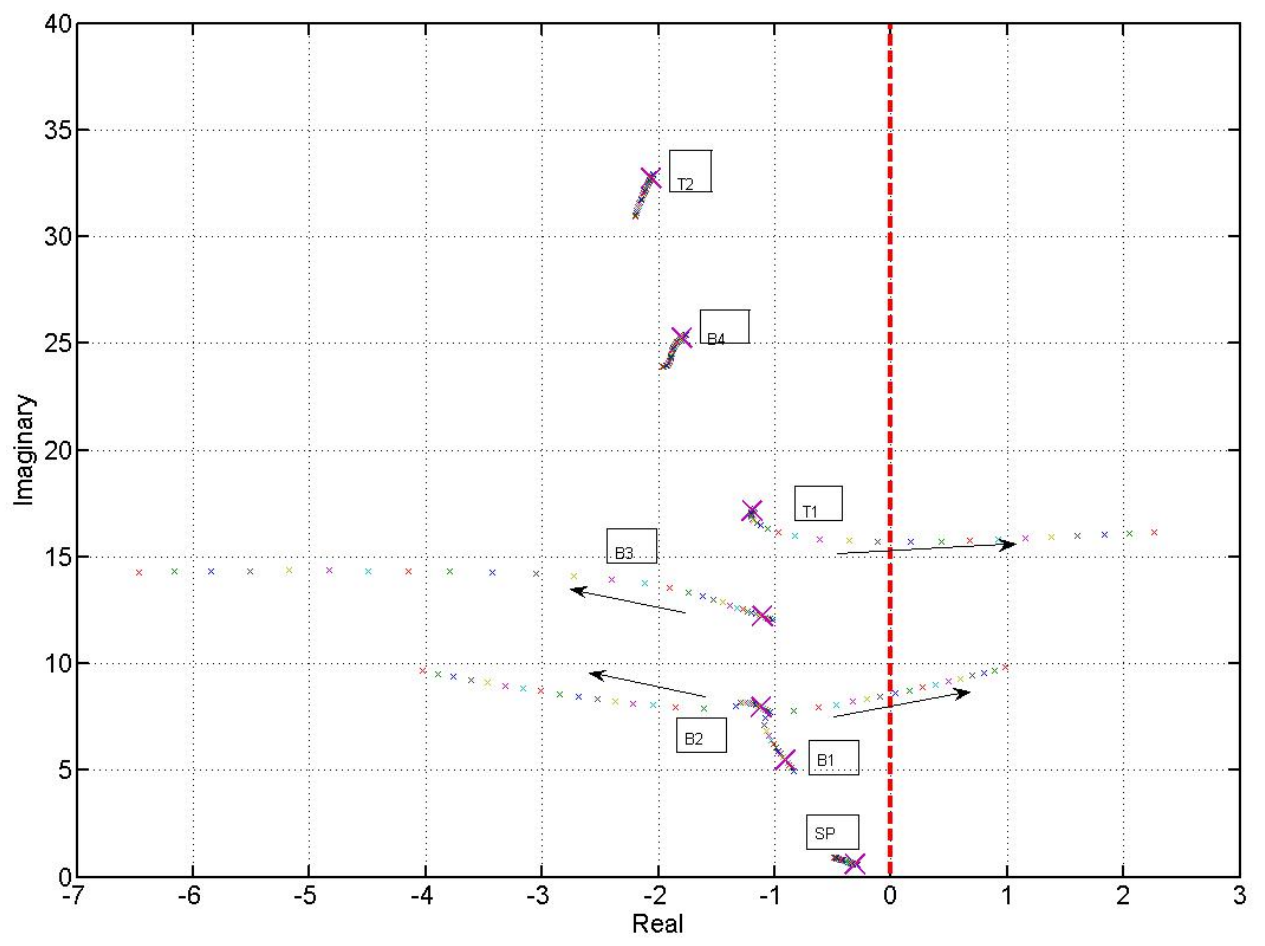

Figure 12. Root locus as air speed varies from Mach 0.6 to 0.9

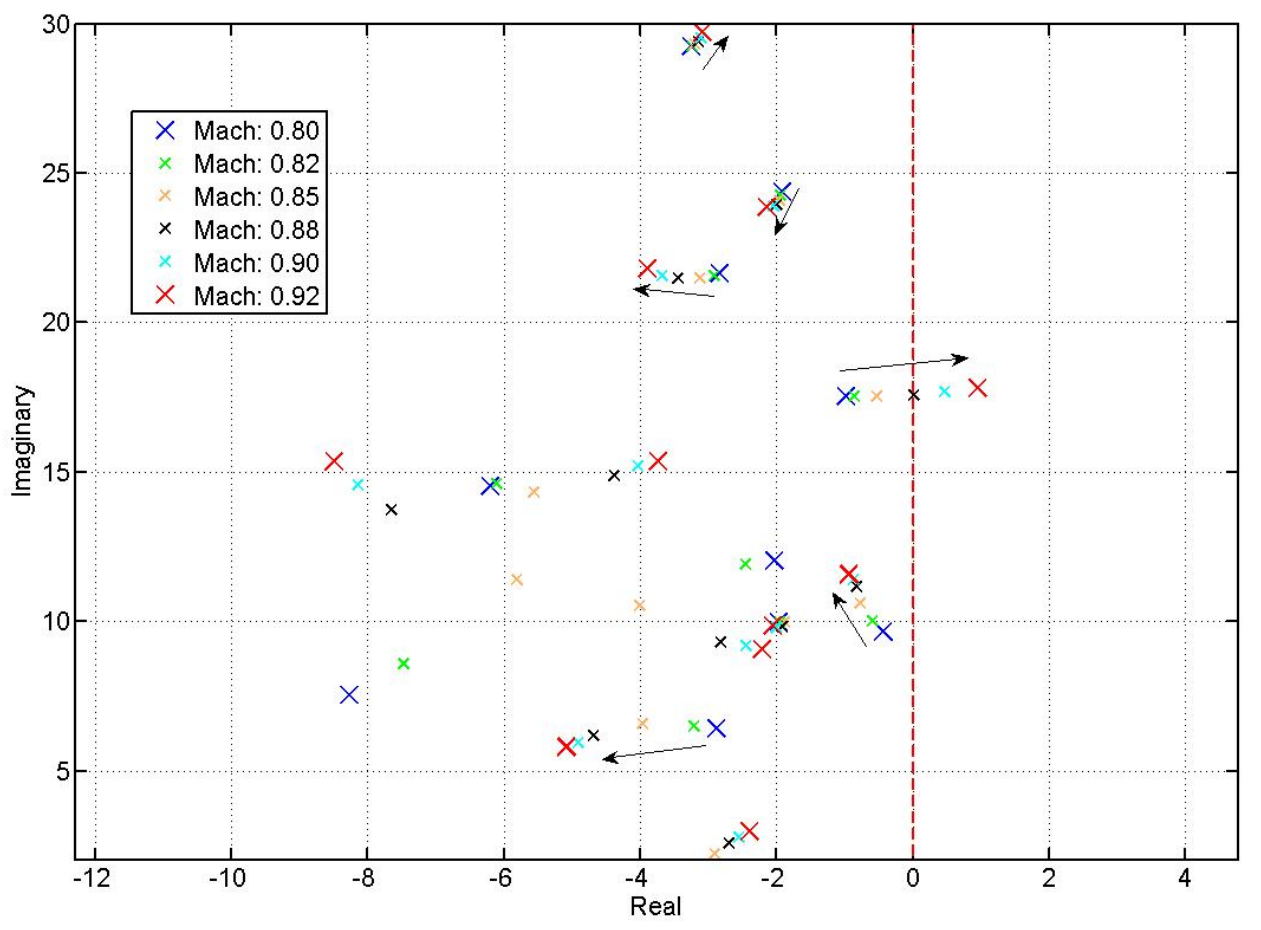

Figure 13. Closed-loop root locus as air speed varies from Mach 0.8 to 0.92 


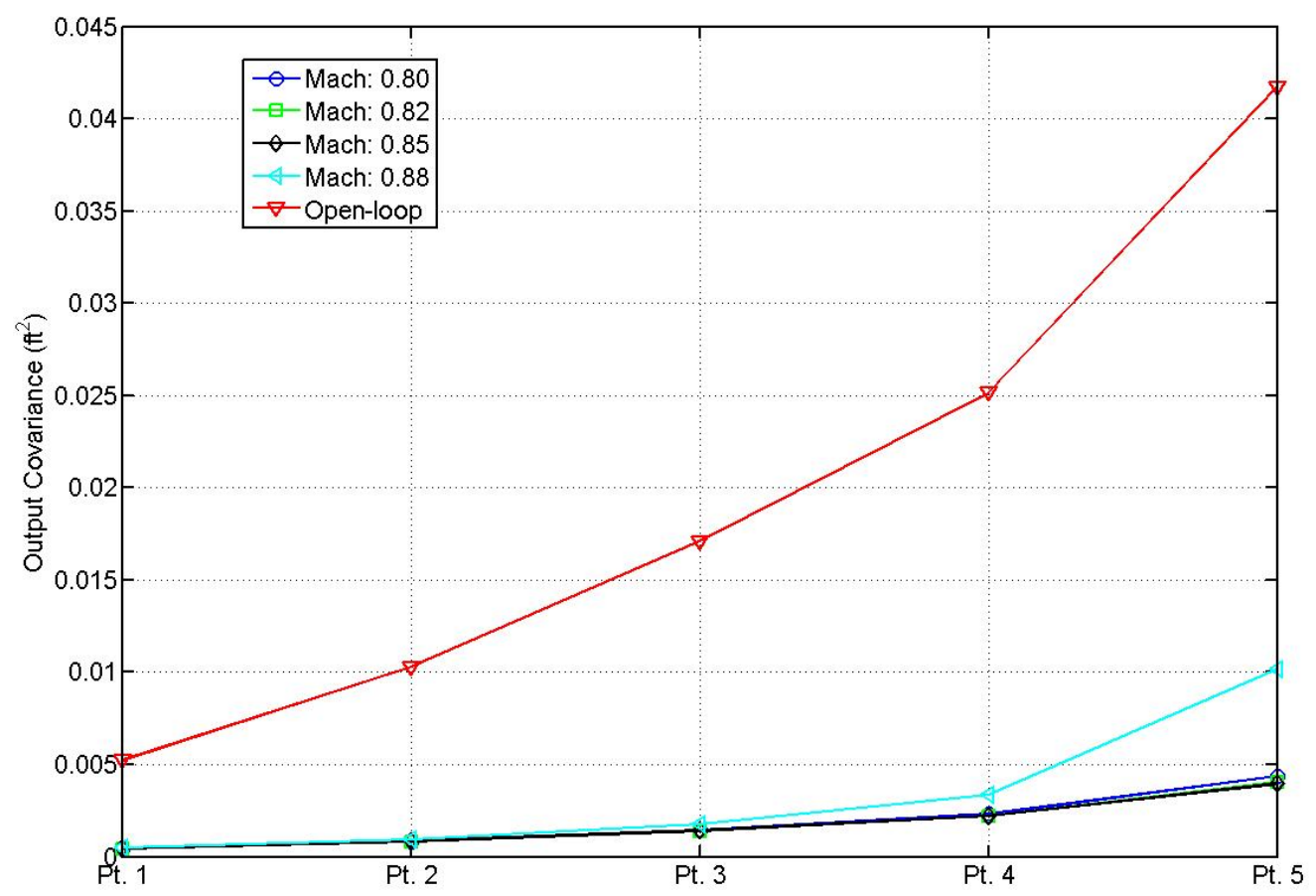

Figure 14. Closed-loop output covariance (bending) comparisons

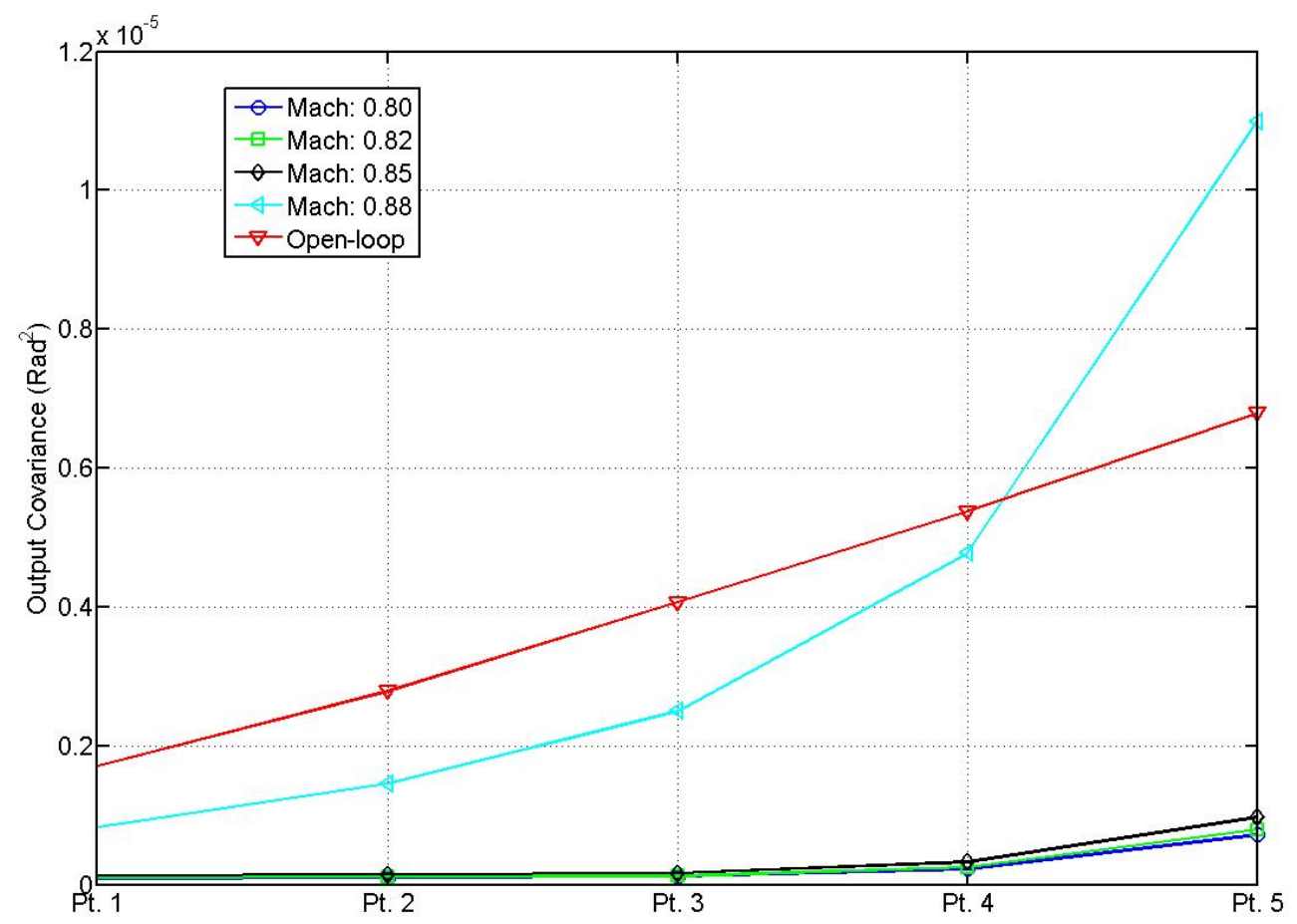

Figure 15. Closed-loop output covariance (torsion) comparisons 